\title{
Changements sociaux et dynamiques politiques
}

Jean-Pierre Jessenne et Hervé Leuwers

\section{(2) OpenEdition}

Journals

Édition électronique

URL : https://journals.openedition.org/ahrf/11461

DOI : 10.4000/ahrf.11461

ISSN : 1952-403X

Éditeur :

Armand Colin, Société des études robespierristes

Édition imprimée

Date de publication : 1 janvier 2010

ISSN : 0003-4436

Référence électronique

Jean-Pierre Jessenne et Hervé Leuwers, "Changements sociaux et dynamiques politiques », Annales historiques de la Révolution française [En ligne], 359 | janvier-mars 2010, mis en ligne le 17 mars 2011, consulté le 23 avril 2022. URL : http://journals.openedition.org/ahrf/11461 ; DOI : https://doi.org/ 10.4000/ahrf.11461 


\title{
CHANGEMENTS SOCIAUX ET DYNAMIQUES POLITIQUES
}

\author{
Jean-Pierre JESSENNE \\ Hervé LEUWERS
}

La mise en relation complexe des changements sociaux et des dynamiques politiques pourrait être admise comme le paradigme essentiel de l'histoire de toutes les révolutions. Pourtant celle de la Révolution française a, sans doute plus que d'autres, donné lieu à une sorte d'oscillation historiographique accentuée entre les priorités interprétatives. Ainsi, et en bref, après que le deuxième tiers du $\mathrm{xx}^{\mathrm{e}}$ siècle eût été marqué par la vigueur dominante de l'explication socio-économique du bouleversement, notamment par sa nature "bourgeoise », de la fin des années 1960 aux années 1980, en un temps de mutations de la discipline, de renouveau de l'histoire politique, d'émergence de l'histoire culturelle et de luttes idéologiques, les visions antagonistes se sont durement confrontées jusqu'à ce que l'histoire politique et culturelle ait semblé l'emporter au temps du Bicentenaire de 1789. Tellement qu'à ses lendemains, Michel Vovelle intitulait son introduction aux communications du Congrès international des Sciences historiques de 1995 : « Du tout social au tout politique $»^{1}$. Dans un rapide et brillant parcours au travers des déclinaisons changeantes du rapport entre histoires sociale et politique de la Révolution depuis la fin du XIX ${ }^{\mathrm{e}}$, il dressait le diagnostic suivant : « Histoire braudélienne, histoire marxiste, quelles que fussent leurs différences, font les frais de cette redistribution des rôles qui, au-delà de l'apparence [...] d'un émiettement du champ historique désormais rétif aux hypothèses globalisantes, laisse

(1) Michel Vovelle, « Du tout social au tout politique », introduction aux communications présentées à la session de la Commission Internationale d'histoire de la Révolution française du Congrès international de Sciences historiques (Montréal, 1995), AHRF, n 310, 1997-4, p. 545-554. 
apparaître l'hégémonie nouvelle de l'histoire des représentations au sein desquelles la dimension politique tend à s'autonomiser à une place singulière ». Pour autant, Michel Vovelle continuait à voir dans la Révolution française « un champ d'expérimentation exceptionnel, à la rencontre du social et de l'émergence du politique, à travers le mouvement des idées, les pratiques inventées, l'imaginaire et le symbolique qui s'y déploient, le conflit enfin entre systèmes de représentations antagonistes dont elle a été le lieu privilégié $\gg{ }^{2}$.

Champ d'expérimentation, là est sans doute effectivement l'essentiel qui permet de sortir de la tentation dichotomique séparant trop schématiquement le social et le politique; l'historiographie récente y invite avec quelques balbutiements. Certes, les débats ne sont pas clos; ces dernières années, ils ont notamment été relancés par les conclusions divergentes des ouvrages d'Henri Heller, prônant une lecture d'inspiration marxiste de la révolution bourgeoise, et celui de Sarah Maza, ramenant la bourgeoisie française au rang de mythe ${ }^{3}$. Il est cependant désormais possible de les aborder différemment, et le récent colloque Vers un ordre bourgeois a ainsi permis de procéder à un bilan international, par le prisme d'une interrogation sur la nature bourgeoise des acteurs et sur l'embourgeoisement de la société révolutionnée ${ }^{4}$. Si la question de la dimension sociale de la Révolution reste au centre des interrogations, ses approches ont la diversité de l'historiographie de notre temps, récemment enrichie des apports des gender studies ou du renouveau de l'histoire coloniale. La décennie passée a en somme confirmé, enrichi et diversifié les pratiques de recherche qui assignent à l'histoire une position singulière dans les sciences humaines, fondée sur la mise en relation de toutes les dimensions de la vie en société et notamment des enjeux sociaux et des évolutions politiques. Cette évidente vitalité s'est manifestée par des approches diverses dont les colloques-bilans de 2003 et 2004 ont donné d'efficaces aperçus $^{5}$. Il ne s'agit pas ici d'esquisser un nouveau bilan et moins encore

(2) Ibidem p. 554

(3) Henry Heller, The Bourgeois Revolution in France, 1789-1799, New York, Barghahn books, 2006; Sarah MAzA, The Myth of the French Bourgeoisie : An Essay on the Social Imaginary, 1750-1850, Cambridge, Harvard University Press, 2003.

(4) Jean-Pierre JESSENNE (dir.), Vers un ordre bourgeois? Révolution française et changement social, Rennes, PUR, 2007.

(5) Martine Lapied, Christine Peyrard (dir.), La Révolution française au carrefour des recherches, Aix-en-Provence, Publications de l'Université de Provence, 2003; Jean-Clément MARTIN (dir.), La Révolution à l'ouvre. Perspectives actuelles dans l'histoire de la Révolution française, Rennes, Presses universitaires de Rennes, 2005. 
de prétendre en présenter toutes les dimensions ; l'objet de ce numéro spécial n'a d'autre ambition que de proposer quelques situations ou regards qui croisent construction sociale et dynamique politique dans trois directions principales.

La première relève de ce qu'on peut appeler l'histoire sociale des institutions ou des pouvoirs. Pour la Révolution, il s'agit d'une vue dialectique qui associe l'étude des fonctions et des organismes - souvent neufs créés par la loi à l'examen du recrutement de ceux qui les investissent, de leurs comportements ou de leurs stratégies, mais aussi les effets performatifs de ces institutions sur la vie sociale au travers de l'élaboration de nouvelles catégories d'analyse ${ }^{6}$. Ainsi, les travaux prosopographiques sur le recrutement et le fonctionnement des assemblées législatives, les études consacrées à certains protagonistes de la Révolution comme les représentants en mission ou le large investissement à différentes échelles de l'exercice des pouvoirs locaux constituent des modes très productifs de conjugaison des histoires sociale et politique de la Révolution ${ }^{7}$.

La connexion entre les deux s'avère plus explicite encore dans un type d'étude exemplaire de l'historiographie récente, celle qui s'attache à décrypter les ressorts enchevêtrés de l'action politique : le travail de Timothy Tackett cherchant à comprendre comment les Constituants deviennent révolutionnaires, celui d'Haïm Burstin révisant la manière dont les protagonistes révolutionnaires du faubourg Saint-Marcel endossent la figure du sans-culotte ou celui d'Anne de Mathan s'interrogeant sur le profil social, économique et culturel des « girondistes » bordelais procèdent de cette démarche ${ }^{8}$. À certains égards, quand Roger Dupuy prolonge les réflexions des années 1980 sur la culture politique populaire en recherchant les manifestations de « la politique du peuple », il procède par d'autres voies au même genre d'investigation'.

Une troisième lecture tente d'isoler les conditions et les modalités de l'adaptation des individus ou des groupes sociaux aux nouvelles condi-

(6) Pour une commode mise en perspective, voir Philippe Minard, « Quelle histoire sociale des institutions », Bulletin de la SHMC, 2000/3 et 4, p. 119-123.

(7) Voir notamment: Edna H. Lemay (dir.), Dictionnaire des Législateurs, 1791-1792, Ferney-Voltaire, Centre international d'étude du XVIII ${ }^{\mathrm{e}}$ siècle, 2007, 2 vol. Michel BIARD, Missionnaires de la République. Les représentants du peuple en mission (1793-1795), Paris, Editions du CTHS, 2002.

(8) Timothy Tаскетt, Par la volonté du peuple. Comment les députés de 1789 sont devenus révolutionnaires, Paris, Albin Michel, 1997. Haïm Burstin, Une révolution à l'œuvre : le faubourg Saint-Marcel (1789-1794), Seyssel, Champ Vallon, 2005. Anne DE MATHAN, Girondins jusqu'au tombeau. Une révolte bordelaise dans la Révolution, Bordeaux, Editions du Sud-Ouest, 2004.

(9) Roger Dupuy, La politique du peuple, Paris, Albin michel, 2002. 
tions sociales, économiques ou politiques, par-delà la Révolution. Il peut s'agir d'une interrogation sur des personnages, ou sur des dynasties; à plus d'un titre, l'étude consacrée par Michel Zylberberg à la famille Le Couteulx reste à cet égard exemplaire ${ }^{10}$. Il peut également s'agir d'une interrogation sur les mutations d'un groupe aux frontières plus ou moins floues, tel celui des bourgeois de Paris étudiés par David Garrioch ou ces « élites grenobloises » récemment suivies par Sylvain Turc des Lumières à la monarchie de Juillet ${ }^{11}$. D'autres encore, par une démarche plus interprétative et globalisante se réclamant des travaux ayant renoncé à chercher un agent unique de l'histoire révolutionnaire, se focalisent sur un processus jugé décisif dans les changements sociaux et politiques mêlés. C'est notamment la voie ouverte par Colin Jones qui multiplie les contributions pour montrer l'intensification des échanges et la commercialisation des rapports sociaux dans la France du XVIII ${ }^{\mathrm{e}}$, comme contexte dans lequel s'inscrit la Révolution ${ }^{12}$.

Chacun à sa manière, ces travaux ont permis la mise en forme d'une nouvelle histoire socio-politique du passage des temps modernes à l'époque contemporaine, que ce numéro souhaite accompagner et encourager. Issus de générations et d'horizons historiographiques divers, les auteurs sollicités n'ont pas eu pour but de s'inscrire explicitement dans l'un ou l'autre des champs ci-dessus évoqués, mais plutôt d'aborder, chacun à sa manière, deux questions clés qui les traversent presque tous. La première rejoint, a priori, les grandes controverses des années 1970 et 1980, et il peut paraître provocateur de poser une nouvelle fois la question du lien entre appartenance sociale et action politique. Le renouveau des études biographiques et prosopographiques sur les acteurs sociaux et politiques, sur les membres des réseaux de sociabilité, n'ont-ils pas démontré l'imparfaite adéquation entre ces deux éléments et la complexité des réponses possibles? Sans nier ces éléments, comment cependant écrire l'histoire du politique sans une réflexion sur les acteurs, leurs motivations, leurs attentes, les modalités de leur action ou les conditions d'élaboration d'une conscience révolutionnaire? S'interroger sur les engagements ou

(10) Michel Zylberberg, Capitalisme et catholicisme dans la France moderne. La dynastie Le Couteulx, Paris, Publications de la Sorbonne, 2001.

(11) David Garrioch, The Formation of the Parisian Bourgeoisie (1690-1830), Cambridge \& London, Harvard University Press, 1996. Sylvain TuRc, Les élites grenobloises des Lumières à la monarchie de Juillet, Grenoble, PUG, 2009.

(12) Voir au point de départ de ces analyses la contribution programmatique «Bourgeois Revolution revivified : 1789 and Social Change » dans Colin LuCAS (ed), Rewriting the French Revolution, Oxford, 1991, p. 69-118. 
actions politiques socialement identifiables, sur les conditions dans lesquelles ils prennent corps, n'est pas nier la diversité du social, mais tenter de le rendre intelligible. La seconde question, quant à elle, entend revenir sur les processus de construction sociale en période de trouble politique, et la manière dont les groupes se redéfinissent et se donnent à voir, particulièrement au moment de la Révolution et de l'Empire; les évolutions les plus fondamentales, provoquées par la fin de la féodalité ou la réforme judiciaire sous la Révolution, par le renouveau des professions médicales ou la réinvention de la noblesse sous l'Empire viennent immédiatement à l'esprit et disent l'importance des changements à l'œuvre. Ensemble, elles invitent à une écriture sociale et politique de l'histoire de la Révolution et de l'Empire.

Jean-Pierre JESSENNE Hervé LEUWERS

UMR IRHiS - 8529 CNRS, Université Lille 3

BP 60149

59653 Villeneuve-d'Ascq

jean-pierre.jessenne@univ-lille3.fr herve.leuwers@univ-lille3.fr 